\title{
Subungual Osteochondroma or Exostosis Cartilaginea of the Hallux: A case report of recurrent bone tumor one year later
}

\author{
by Al Kline, $\mathbf{D P M}^{1} \square$
}

The Foot and Ankle Online Journal 3 (2): 3

Osteochondroma is the most common benign bone tumor of the foot. This case describes a teenage girl who returns one year later with recurrent osteochondroma. The etiology, diagnosis and treatment options of this tumor are discussed. After initial excision, a revisional surgery was performed one year later that included more aggressive curettage of the recurrent osteochondroma with allogenic bone grafting using InterGro ${ }^{\circledR}$ DBM Plus.

Key words: Subungual tumor, osteochondroma, subungual exostosis.

Published: February, 2010

This is an Open Access article distributed under the terms of the Creative Commons Attribution License. It permits unrestricted use, distribution, and reproduction in any medium, provided the original work is properly cited. @The Foot and Ankle Online Journal (www.faoj.org)

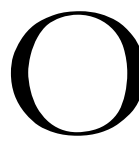
steochondromas are small, benign, bone neoplasms and are the most common bone tumor of the foot. ${ }^{1,2}$ In the foot, they are most commonly found at the end of small bones, such as the phalanx of the toes. Osteochondromas are similar to the subungual exostosis under the hallux nail plate. It is termed 'Exostosis Cartilaginea' because of its distinctive cartilaginous cap as the tumor protrudes through the nail bed. Typically, the bone originates from the metaphyseal surface of long bones and is capped by growing cartilage. ${ }^{2}$ In recent studies, there is now increasing evidence of histological differences between subungual exostosis and subungual osteochrondroma. Histologically, the subungual exostosis has a fibrocartilage cap whereas the osteochondroma has distinctive hyaline cartilage. ${ }^{1}$

Address correspondence to: Al Kline, DPM

3130 South Alameda, Corpus Christi, Texas 78404.

Email: alklinedpm@gmail.com.

${ }^{1}$ Adjunct Clinical Faculty, Temple University School of Podiatric Medicine, Barry University School of Podiatric Medicine. Private practice, Chief of Podiatry, Doctors Regional Medical Center. Corpus Christi, Texas, 78411.
Solitary osteochondromas account for 35 to 40 percent of all benign bone tumors. ${ }^{3,4}$ Most tumors are found in patients younger than 20 years of age. The male-to-female ratio is $3: 1^{4}$ However, in the subungual tumor, there is no strict sex ratio. ${ }^{1}$

An isolated osteochondroma is usually associated with trauma, especially in the distal phalanx of the hallux. Although in general, the cause is not clearly understood or known it has also been reported as congenital. However, trauma appears to be a more commonly reported finding in the subungual tumor. The tumors may develop abnormally with or without a stalk. When the stalk is present, the tumor is termed pedunculated. When the stalk is absent, it is called sessile. ${ }^{4}$ Osteochondromas rarely become malignant and mitotic figures are rarely identified microscopically. In less than $1 \%$ of all solitary osteochondromas, malignant degeneration of the cartilage cap is usually heralded by new onset of growth, pain and rapid growth of the lesion. ${ }^{4}$ 


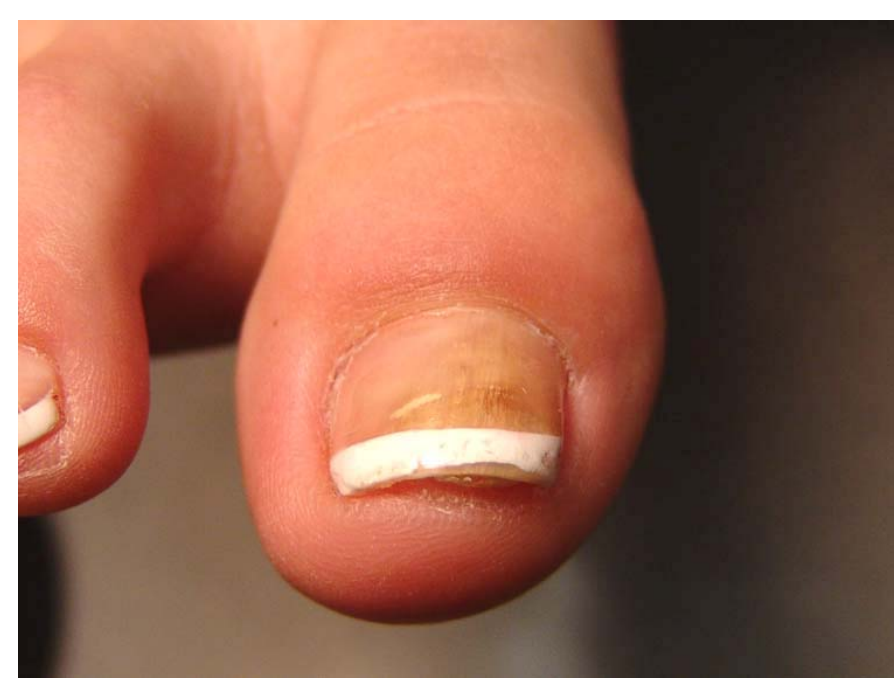

Figure 1 A 15 year-old female presents with painful right hallux nail. She is active in volleyball and track. The nail plate has distinctive color changes and partial onycholysis of the nail plate. Her initial concern was possible 'toenail fungus' and 'pain'.

The most commonly reported transformation is to a chondrosarcoma. The potential for malignant transformation occurs more often in the hereditary disorder called hereditary multiple cartilaginous exostoses. Malignant transformation to osteogenic sarcoma has also been reported, but not in solitary, subungual osteochondromas. ${ }^{2}$ As a subungual tumor, the lesion usually protrudes up through the soft nail bed and appears as firm, slightly lobulated, marblelike or sometimes translucent cartilaginous bone that causes onycholysis of the nail plate. It can appear strikingly similar to enchondromas and glomus tumors. ${ }^{1}$ On gross examination, enchondromas also have a cartilaginous cap and appear lobulated, glassy, gray-blue and translucent as the tumor erodes through cortical bone. ${ }^{2}$ Unlike osteochondromas that originate from the metaphyseal surface of the bone, enchondromas occur deep within the spongiosa of bone. ${ }^{2}$ Glomus tumor or glomangioma is a common benign tumor of the fingers and toes that manifest under the nail. Glomangiomas are supplied with an efferent artery, AV anastomoses and efferent veins measuring about $5 \mathrm{~mm}$ in diameter on average. This gives the lesion nodular, firm consistency and its distinctive red-blue color.

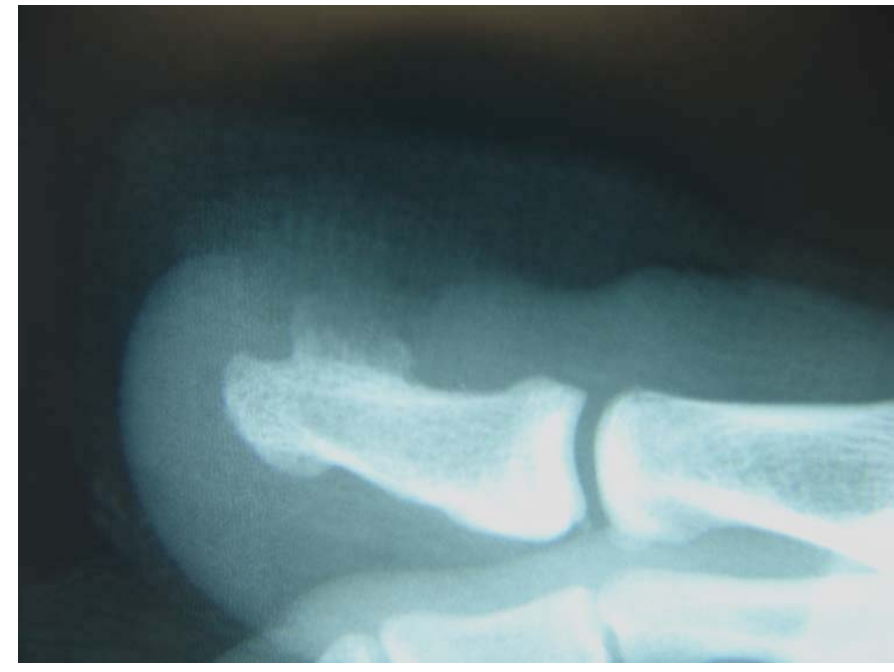

Figure 2 Hallux views reveal a large subungual exostosis arising from the metaphyseal portion of the distal phalanx.

Differential diagnosis of osteochondroma includes enchondroma, pyogenic granuloma, verruca vulgaris, lipoma, nonossifying fibroma, glomangioma, simple bone cyst, unicameral bone cyst, fibrous dysplasia, eosinophilic granuloma, chondroblastoma, chondromyxoid fibroma, osteogenic sarcoma or clear cell chondrosarcoma. ${ }^{1,2,3,4}$ A case report of a young girl is presented. She initially presented with a typical, subungual osteochrondroma which was surgically removed. One year following her surgery, she presented to our office with recurrence of the tumor.

\section{Case Report}

A 15 year-old female presented to our office on February 1, 2006 with pain to the right hallux. She was also concerned that the nail was 'discolored' with a possible fungal infection. (Fig. 1).

The patient was very active in volleyball and track. She states that her toe was now "throbbing" during her activities. She also recalled sustaining a more acute injury during a volleyball game a few months prior to presentation. Radiographic evaluation on the lateral hallux view revealed a large, subungual like exostosis. The exostosis appeared to be originating from the metaphyseal surface of the phalanx. (Fig. 2) 


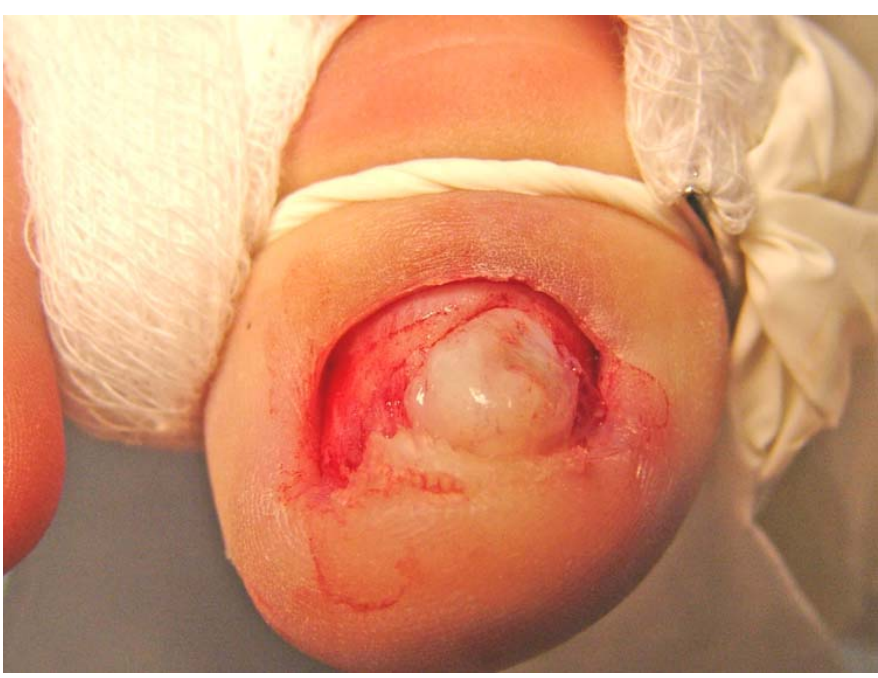

Figure 3 Removal of the nail plate reveals a lobulated, irregular, firm nodule with a distinctive translucent cartilaginous cap. Differential diagnosis include osteochondroma, enchondroma, chondroblastoma or other tumors of cartilaginous origin.

The patient was scheduled for surgical removal of the exostosis. When the nail was avulsed, a large, lobulated, firm nodule with distinctive, translucent cartilaginous cap was identified. (Fig. 3)

The lesion was simply removed by simple exostectomy. A rongeur was used to remove the lesion and a small depression was made in the bone. The surrounding soft tissue nail bed was gently placed in the depression and then dressed with Adaptic ${ }^{\mathrm{TM}}$ and cotton-gauze dressing. The patient was sent home and placed in a Darco ${ }^{\mathrm{TM}}$ shoe for about 2 weeks. A few days after the procedure, her dressings were removed. The nail bed already revealed signs of tissue healing. (Fig 4) The nail bed healed and she returned to full activity about 2 months after the procedure.

\section{Histology}

The initial gross specimen was received in formalin measuring $1.5 \times 1.3 \times 0.3 \mathrm{~cm}$ in aggregate. Microscopic evaluation revealed subungual skin showing psoriasiform squamous epithelial hyperplasia.

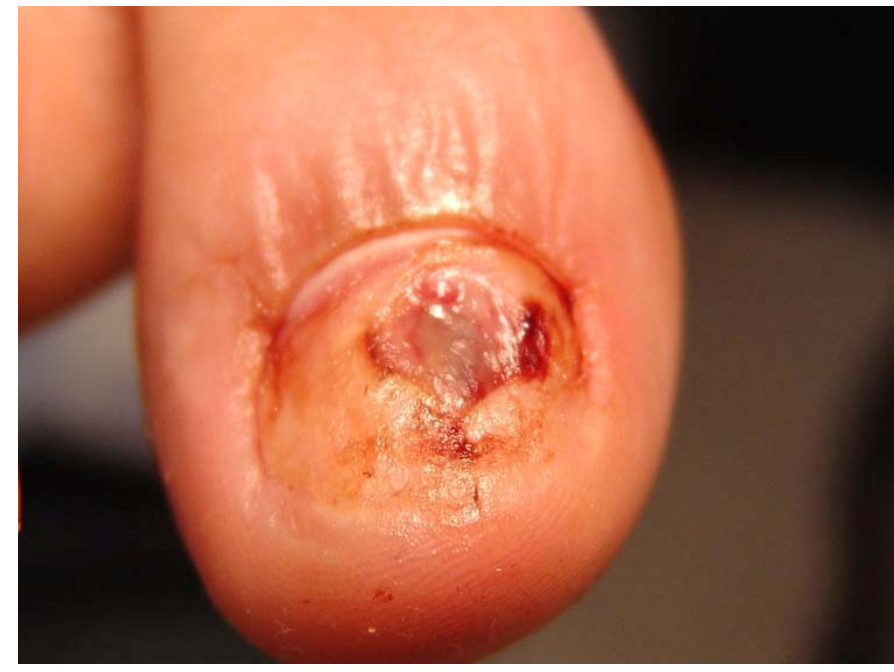

Figure $4 \mathrm{~A}$ few days after the excision of the tumor, healing of the nail bed is seen.

Adjacent area was present containing fibroblastic and fibrohistiocytic cellular elements. Plump fibroblasts were detected, accompanied by multi-nucleated giant cells in a background of patchy chronic inflammation. The fibroblastic zone contained small fragments of devitalized mature lamellar bone. Other fields showed reactive or woven bone.

The case report and radiographs were sent to Dr. Alberto Ayala, formerly at M.D. Anderson Cancer Center and now at the Methodist Hospital in Houston, Texas for review. The consultation report revealed fragments of bone remarkable for the presence of actively growing osteocartilaginous cap that was forming bone on a cartilaginous background. There was significant granulation tissue with infiltration by acute and chronic inflammatory cells. In the opinion of this consultant, the lesion was a subungual osteochondroma which is sometimes referred to as "traumatic osteochondroma" with associated secondary inflammatory changes. The report read "This type of lesion is commonly seen in the toe and most cases have a similar history of trauma as in this patient."

(C) The Foot and Ankle Online Journal, 2010 


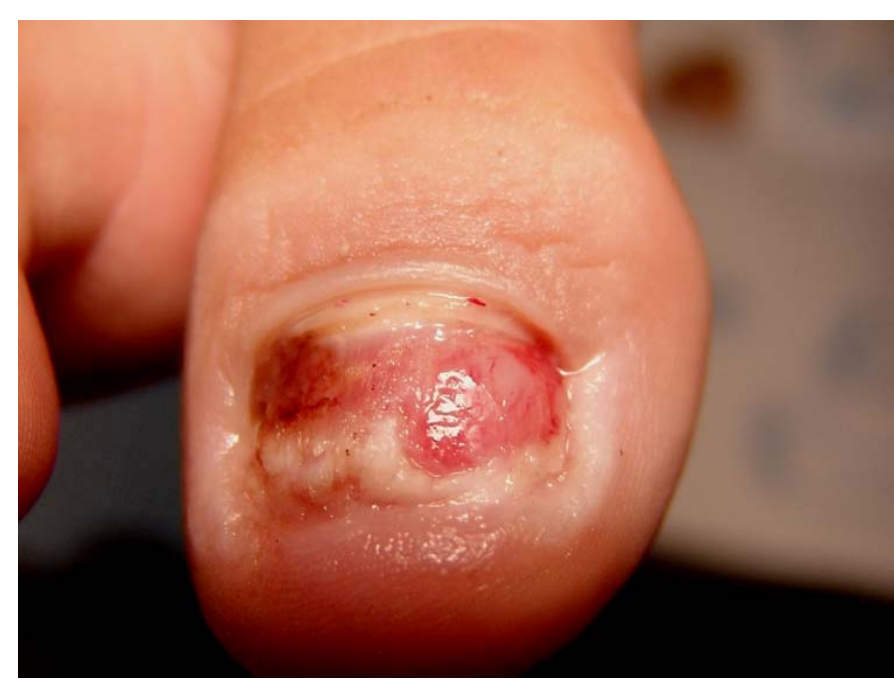

Figure 5 The patient presented almost 1 year later with similar, recurrent pain to the hallux. Inspection of the hallux reveals nail bed changes were consistent with recurrent tumor in the exact location as the previous excision.

\section{Recurrence of the Tumor}

The patient returned in late 2006 with complete regrowth of the nail plate. The nail continued to be thick. In early February 2007, the patient re-presented to our office with recurrent pain almost 1 year to the day of her previous exostectomy. The nail appeared loose with attachment proximally at the epinychial nail fold. The patient reported the nail 'fell off'. (Fig. 5) Radiographs were ordered which revealed a small, recurrent region of exostosis from the metaphyseal portion of bone in the same location as the previous tumor. (Fig. 6) The patient was then scheduled for a more aggressive resection of the tumor.

\section{Surgical Technique}

We decided that saucerization with high speed burr and allogenic bone grafting would be attempted to prevent a tertiary recurrence. The patient was brought to the operating room. Under local hallux block, the entire tumor was dissected and the dorsal cartilaginous cap was removed en-bloc excision. (Fig. $7 \mathrm{~A})$

(C) The Foot and Ankle Online Journal, 2010

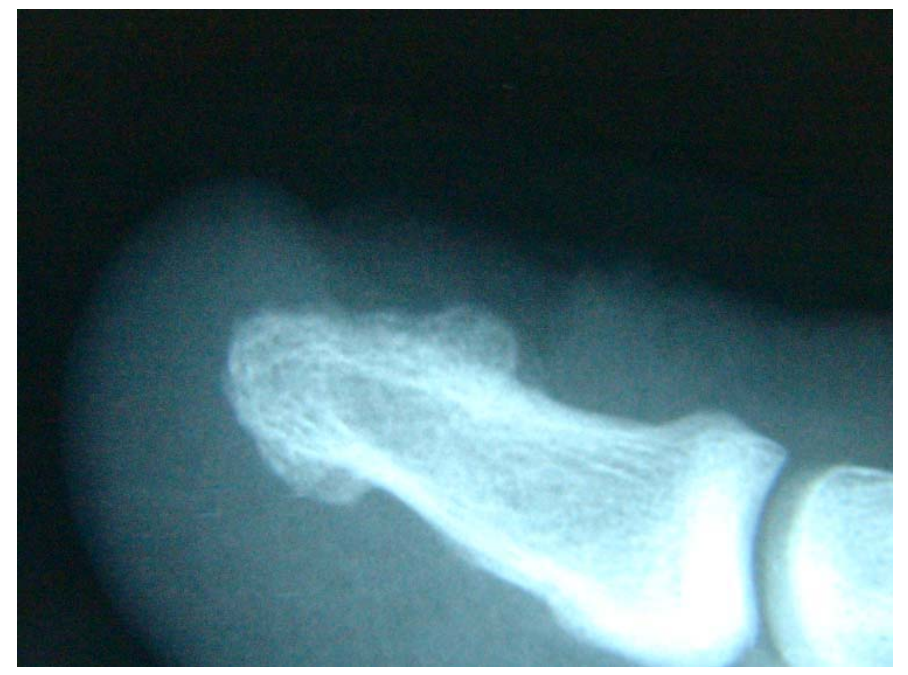

Figure 6 Recurrent bone growth was seen in the exact location as the previous tumor that was resected 1 year prior.

The underlying bone was identified. A high-speed burr was then used to saucerize the region. (Fig. 7B) Care was taken to not disrupt the proximal nail matrix or surrounding soft tissue nail bed. The defect was then curettaged of residual bone fragments. (Fig. 7C)

Once the defect was made, a small burr was used to punch 'holes' into the underlying cancellous bone structure. The defect was now ready for bone grafting. A small Freer elevator was used to impact a small amount of InterGro ${ }^{\circledR}$ DBM Plus bone graft into the defect. (Fig. 8A and 8B)

The DBM or demineralized bone matrices is actually porous calcium granules mixed in a lipid carrier. The graft material is allogenic human tissue that has been granulated for transplantation. EBI ${ }^{\circledR}$, who makes InterGro ${ }^{\circledR}$, reports that the tissue was recovered from deceased donors whose legal next-of-kin have given permission for the bone to be donated. Recovery was performed using sterile procedures and packaging was performed using aseptic techniques in a controlled clean-room environment. The use of this material is contraindicated in patients with incomplete skull growth or in areas of active infection. ${ }^{8}$ InterGro ${ }^{\circledR}$ Plus contains 35 percent DBM by weight. (Fig. 9) 


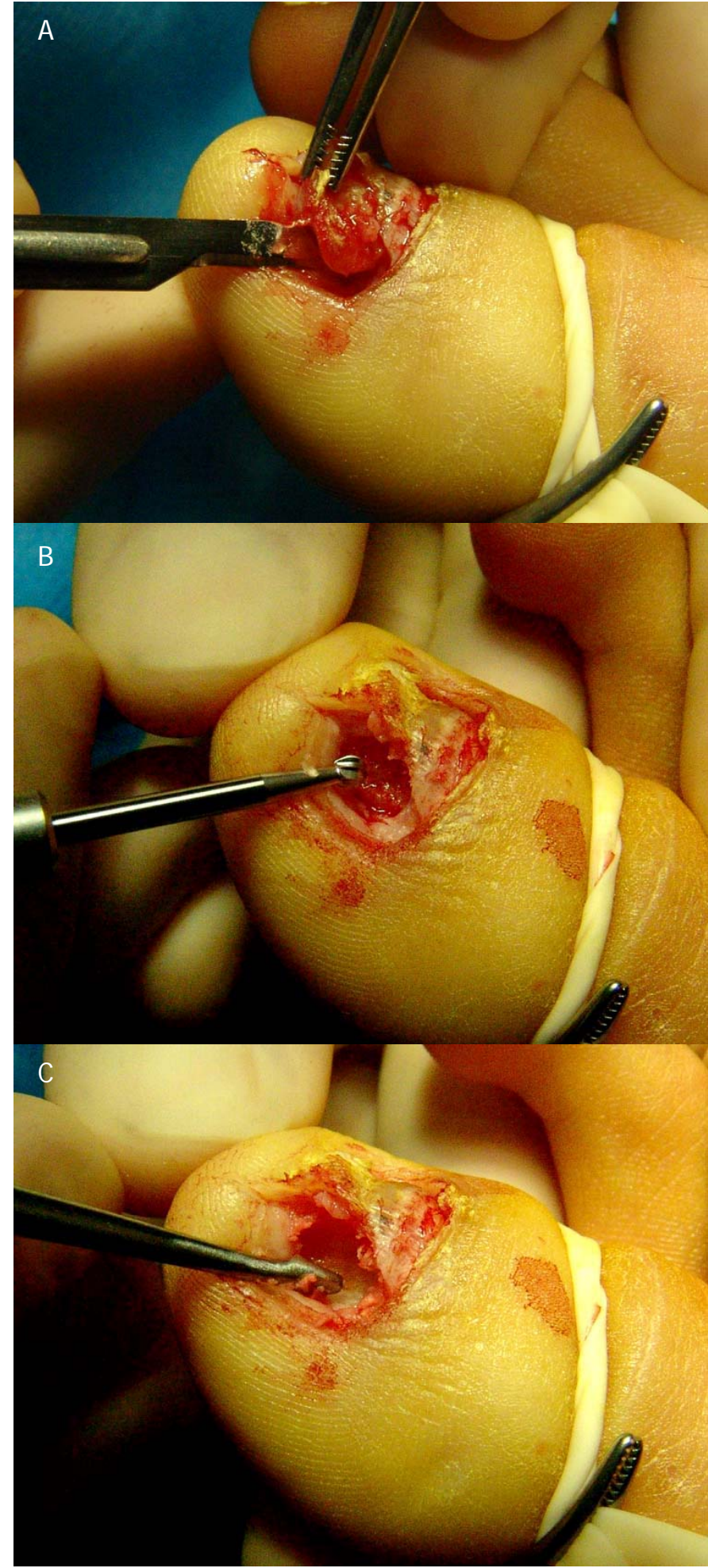

Figure $7 \mathbf{A}$ - C The cartilaginous portion of the tumor was removed en-bloc down to raw bone. (A) A highspeed rotary burr was then used to saucerize and remove the remaining bone. (B) A curette was then used to remove any loose bone from the base of the defect. (C)

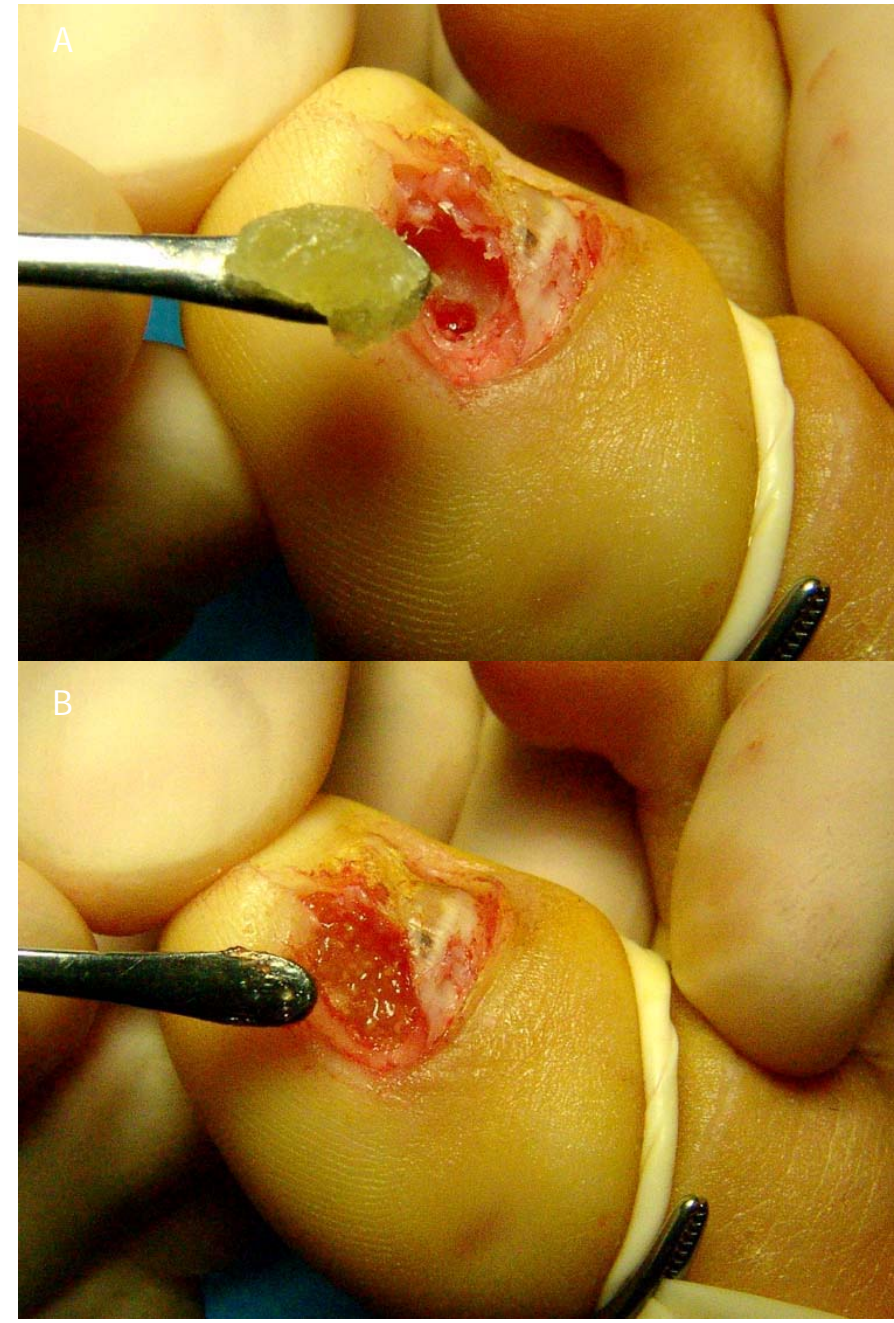

Figure $\mathbf{8 A}$ and $\mathbf{8 B}$ The high-speed burr was used to punch holes in the base of the saucerized bone. InterGro ${ }^{\circledR}$ DBM was then placed into the defect to fill the space. The region was then dressed with Adaptic ${ }^{\text {TM }}$ gauze and cotton ball dressing.

\section{Discussion}

Osteochondroma appear to be a common benign tumor most commonly described in the foot as a subungual lesion. The tumor has also been reported 'extraskeletal' within the foot, but is exceedingly rare. ${ }^{5}$ The recurrence rate after resection of osteochondroma is about 1.8 percent. ${ }^{4}$ Eliezri and Taylor reported two cases of recurrent subungual osteochondroma, with one recurring just a few weeks after excision. $^{6}$

(C) The Foot and Ankle Online Journal, 2010 


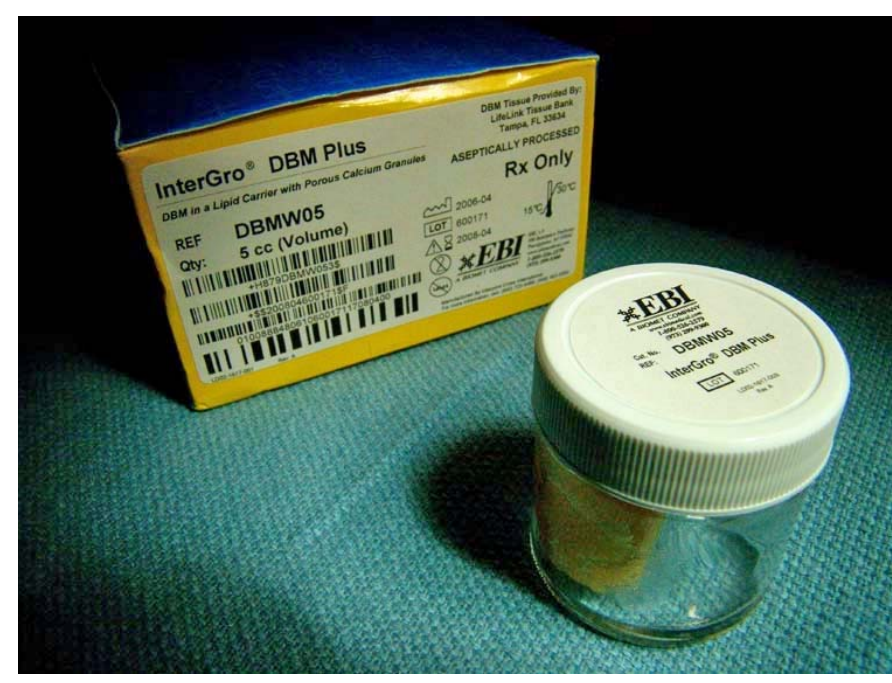

Figure 9 InterGro ${ }^{\circledR}$ is a ProOsteon ${ }^{\mathrm{TM}}$ Osteobiologic product. It contains demineralized, allogenic bone granules in a lipid carrier. These products, produced by $\mathrm{EBI} \circledast{ }^{\circledR}$, contain porous ceramic granules that are a composite of highly resorbable calcium carbonate with a slower resorbing 2 to 10 um outer layer of calcium phosphate. ${ }^{8}$

Recurrence of the tumor is likely caused by cells of the resected perichondrium or cartilage cap that is left behind in unresected bone or nail bed. During the initial removal, it is possible some cells may have been left behind by simple excision without aggressive curettage of bone or adjuvant procedures to destroy the perichondrium.

It could also be the result of persistent or repetitive trauma to the phalanx post resection. The patient was very active and did continue to play volleyball and participate in track. It is interesting to note that in the report of two cases by Tuzuner, et al., there was no incident of trauma associated with those cases. They reasoned that since there was no history of trauma in those two cases, it may be suggested that "a neglected trauma to an immature bone may result in subungual osteochondroma." Both patients were male at 30 and 15 years of age respectively. Histologically, their specimens revealed "classic histopathologic finding of osteochondroma" as a "well-defined trabecular bone covered with a byaline cartilaginous cap."
Tuzuner et, al., also introduced an important distinction between subungual exostosis and osteochondroma. ${ }^{1}$ However, our histologic findings revealed some similarities to subungual exostosis, but were histologically more consistent with osteochondroma.

In a study by Kinoshita, et al., in a review of 83 cases of bone and soft tissue tumors of the foot, the osteochondroma was the most commonly reported benign tumor. They also reported a more common occurrence of the tumor in females under the age of 19 years. $^{7}$

It is the author's opinion that since the tumor recurred, it is best to initially treat any subungual lesion aggressively by saucerization rather than simple exostectomy.

Treatment options can include ablation, cauterization and the use of allogenic or autogenous bone graft following curettage of the lesion. In this case, it was decided to saucerize and curettage the lesion with a high-speed burr and pack the area with allogenic bone graft. To date, no recurrence has been reported.

\section{References}

1. Tuzuner T, Kavak A, Parlak AH, Ustundag N: Subungual osteochondroma. JAPMA 96 (2): 154 - 157, 2006.

2. Robbins SL, Kumar V: Osteochondroma. Basic Pathology. 4th ed.

W.B. Saunders. 711, 1987.

3. American Academy of Orthopaedic Surgeons:

Osteochondroma (Bone Tumor). July, 2004. Al is this an internet reference?

4. Dickey ID: Solitary osteochondroma. Emedicine, 2004. http://emedicine.medscape.com/article/1256477-overview Accessed $23^{\text {rd }}$ January 2010.

5. Sheff JS, Wang S: Extraskeletal osteochondroma of the foot. JFAS 44 (1): 57 - 59, 2005.

6. Eliezri YD, Taylor SC: Subungual osteochondroma: Diagnosis and management. J Derm Surg Oncol 18: 753 - 758, 1992.

7. Kinoshita G, Matsumoto M, Maruoka T, Shiraki T, Tsunemi K, Futani H, Maruo S: Bone and soft tissue tumours of the foot: review of 83 cases. Jour of Ortho Surg. 10 (2): 173 - 178, 2002. 8. EBI ${ }^{\circledR}$ : InterGro ${ }^{\circledR}$ product insert. 